\title{
A COMPARATIVE STUDY OF DETERMINISTIC AND STOCHASTIC OPTIMIZATION METHODS FOR INTEGRATED DESIGN OF PROCESSES
}

\author{
Mario Francisco ${ }^{1}$, Silvana Revollar ${ }^{2}$, Pastora Vega ${ }^{1}$, Rosalba Lamanna $^{2}$ \\ ${ }^{1}$ Departamento de Informática y Automática. Universidad de Salamanca. Spain. \\ mfs@usal.es; pvega@usal.es \\ ${ }^{2}$ Universidad Simón Bolívar. Dpto. Procesos y Sistemas. Caracas 1081A, Venezuela \\ srevolla@usb.ve; lamanna@usb.ve
}

\begin{abstract}
:
This paper focuses on the application of stochastic (genetic algorithms, simulated annealing) and deterministic (sequential quadratic programming) optimization methods for the Integrated Design of processes considering dynamical non-linear models. Moreover, a hybrid methodology that combines both types of methods is proposed, showing an improvement on performance. Controllability indexes such as disturbance sensitivity gains, the $\mathrm{H}_{\infty}$ norm, and the ISE were considered to obtain optimum disturbance rejection. In order to illustrate and validate our proposal, an activated sludge process with PI schemes is taken. The problem is stated as a multiobjective non-linear optimization problem with non-linear constraints. The application of the mentioned strategies is discussed. The results are encouraging for future application of these techniques to solve synthesis MINLP problems. Copyright (c) 2005 IFAC
\end{abstract}

Keywords: Integrated Design, sequential quadratic programming, genetic algorithms, stochastic optimization, controllability

\section{INTRODUCTION}

Process design has been always a hard task, especially when dealing with chemical process design. Typically, the process engineer determines the economically optimal process configuration among many possible alternatives by making use of heuristic knowledge. After the process flowsheet and design parameters are determined by minimising construction and operation costs, the dynamical behaviour of the system is taken into account for the control system design. Thus, the well established notion that controllability of the process is an inherent characteristic of its design is ignored (Luyben, 1993; Luyben and Floudas, 1994; Vega and Gutiérrez, 1999).

The Integrated Design techniques try to overcome these difficulties, because they integrate controllability issues in the design stage, making the final system more controllable (Luyben, 1993; Luyben and Floudas, 1994; Vega and Gutiérrez, 1999). By using this methodology, plant and control system parameters can be obtained at the same time, and all this parameters provide optimum costs and proper controllability behaviour based on dynamical controllability performance indexes. This problem is stated mathematically as a non-linear multiobjective optimization problem with non-linear constraints including economic, process and control considerations.

Another important issue when designing a plant is the selection of the best configuration for the plant. The problem is then called process synthesis and mathematically involves the use of binary variables, making the optimization more complex to solve. A non-linear MINLP constrained optimization problem is generated and has to be solved (Luyben and Floudas, 1994; Revollar et al., 2004).

Due to the complexity of chemical processes, the classical optimisation methods for solving the process synthesis and Integrated Design, sometimes fail to converge properly because of presence of local minima, discontinuities, numerical problems, etc. For this reason, the study of these optimisation methods and the need to look for alternative ones are important issues to be considered (Kookos and Perkins, 2001; Tsai and Chang, 2001). 
The classical gradient based optimization techniques, such as sequential quadratic programming (SQP), have been broadly applied for constrained optimisation obtaining good solutions in a reasonable amount of computing time (Edgar, 2001; Gill, 1981). However, for complex problems these algorithms sometimes fail to give any solution, and their effectiveness decreases when discontinuities and non convexity are present. In this work, a two steps optimization approach has been proposed to improve SQP algorithm convergence and results.

The stochastic optimisation techniques such as genetic algorithms and simulated annealing (Salamon, 2002; Laarhoven, 1987) are recommended for complex non-linear and discontinuous problems where classical optimisation techniques might fail. This algorithms have been used with good results for this type of problems, and particularly for solving process synthesis (Costa and Oliveira, 2001; Tsai and Chang, 2001; Revollar et al., 2004), but their main drawback is the difficulty to handle constrained problems because the stochastic search operators frequently produce infeasible solutions.

In this paper, a hybrid method for the solution of these complex problems, such as process Integrated Design and synthesis, combining genetic algorithms and SQP to make use of the advantages of both methods. First, the genetic algorithm is good to find candidate solutions close to an optimum, exploring all the search space, without suffering numerical problems, and then these candidate solutions are improved using SQP methods to find a real feasible optimum.

Some results of the application of this hybrid optimization technique to activated sludge Integrated Design are shown in this work. Open and closed loop design with PI control configuration have been studied. A comparison of these results with SQP and stochastic optimisation techniques (genetic algorithms and simulated annealing) is also presented.

\section{DESCRIPTION OF THE PROCESS AND PLANT CONTROLLER}

In order to apply the Integrated Design methodology and compare solutions using different optimization methods, the activated sludge process in a wastewater treatment plant has been chosen.

The plant layout is represented in Figure 1, and it consists of a simple structure with one aeration tank and one secondary settler. The fundamentals of the process are that a microbial population (biomass) living into the bioreactor, transforms the biodegradable pollution (substrate) when dissolved oxygen is supplied through aeration turbines. Water coming out of the reactor goes to the settler, where the activated sludge is separated from the clean water and recycled to the bioreactor.
The process variables are presented in Figure 1. Biomass concentrations are denoted by " $x$ " (mg/l), organic substrate concentrations are denoted by "s" $(\mathrm{mg} / \mathrm{l})$, " $c$ " is used for the oxygen concentrations $(\mathrm{mg} / \mathrm{l})$, and " $q$ " for flow rates $\left(\mathrm{m}^{3} / \mathrm{h}\right)$.

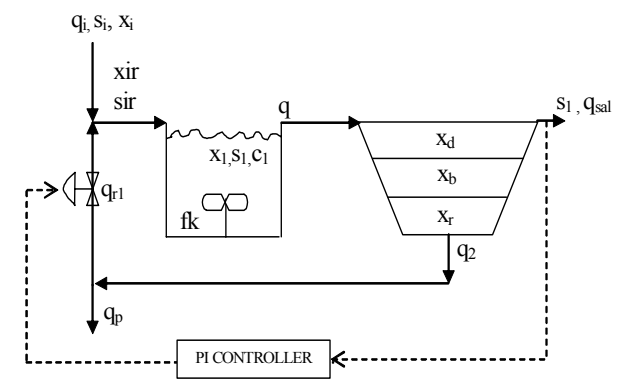

Figure 1: Activated sludge process layout

The control of this process aims to keep the substrate at the output $\left(s_{1}\right)$ below a legal value despite the large variations of the flow rate and the substrate concentration of the incoming water $\left(q_{i}\right.$ and $\left.s_{i}\right)$. These disturbances are one of the main problems when trying to control the plant properly. A conventional PI controller has been considered, with $q_{r 1}$ as manipulated variable. The set of disturbances used as system input in dynamic simulations for designing the plant (Figure 2) has been taken from the European research group COST 624 and its benchmark for wastewater treatment plants.
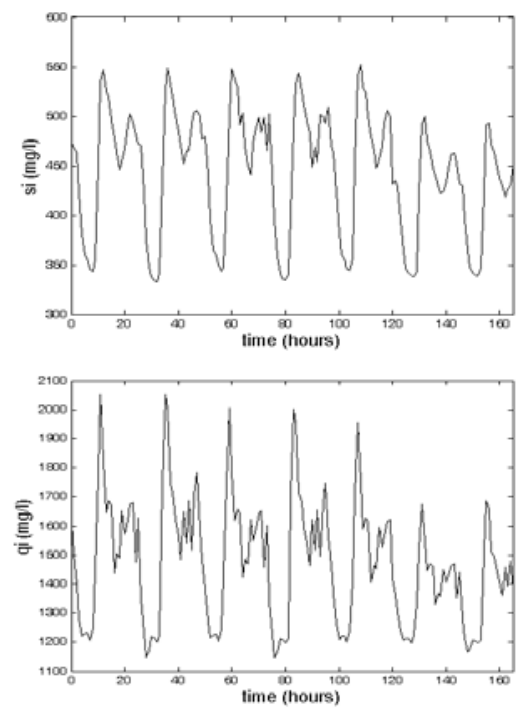

Figure 2: Substrate and flow disturbances at the influent

\section{INTEGRATED DESIGN OPTIMIZATION PROBLEM}

The Integrated Design for the activated sludge process, consists of minimizing an objective function which represents construction and operation costs, while the desired open or closed loop dynamic is considered as constraint. Mathematically it is stated as a NLP/DAE optimization of the following cost function, subject to process and controllability constraints. The problem is formulated as follows: 
Cost function:

$$
f_{1}(x)=w_{1} \cdot V^{2}+w_{2} \cdot A^{2}+w_{3} \cdot f k^{2}+w_{4} \cdot q_{2}^{2}
$$

where $V$ and $A$ are the volume of the reactor and the cross-sectional area of the settler, $f k$ is the aeration factor in the reactor, $q_{2}$ is the total recycling flow and $w_{i}(\mathrm{i}=1, \ldots, 4)$ are the corresponding weights.

Process constraints:

- Residence time and mass load in the aeration tank:

$$
2.5 \leq \frac{V}{q} \leq 8 \quad(2) ; \quad 0.001 \leq \frac{q_{i} s_{i}+q_{r 1} s_{1}}{V \cdot x_{1}} \leq 0.06
$$

- Limits in hydraulic capacity and sludge age in the settler:

$$
\frac{q_{2}}{A} \leq 1.5 \quad(4) ; \quad 3 \leq \frac{V \cdot x_{1}+A l_{r} x_{r}}{q_{p} x_{r} 24} \leq 10
$$

- Limits in the relationship between the input, recycled and purge flow rates:

$$
0.03 \leq \frac{q_{p}}{q_{2}} \leq 0.07 \quad(6) ; \quad 0.5 \leq \frac{q_{2}}{q_{i}} \leq 0.9
$$

- Constraints on the non-linear differential equations of the plant model to obtain a solution close to a steady state ( $\varepsilon$ close to zero):

$$
\begin{aligned}
& \left|\frac{d x_{1}}{d t}\right|=\left|\mu_{\max } y \frac{s_{1} x_{1}}{\left(K_{s}+s_{1}\right)}-K_{d} \frac{x_{1}^{2}}{s_{1}}-K_{c} x_{1}+\frac{q}{V}\left(x i r-x_{1}\right)\right| \leq \varepsilon \\
& \left|\frac{d s_{1}}{d t}\right|=\left|-\mu_{\max } \frac{s_{1} x_{1}}{\left(K_{s}+s_{1}\right)}+f_{k d} K_{d} \frac{x_{1}^{2}}{s_{1}}+f_{k d} K_{c} x_{1}+\frac{q}{V}\left(s i r-s_{1}\right)\right| \leq \varepsilon \\
& \left|\frac{d c_{1}}{d t}\right|=\left|K_{l a} f k\left(c_{s}-c_{1}\right)-K_{01} \mu_{\max } \frac{x_{1}^{2}}{\left(K_{s}+s_{1}\right)}-\frac{q}{V} c_{1}\right| \leq \varepsilon \\
& \left|\frac{d x_{b}}{d t}\right|=\left|\frac{1}{A l_{b}}\left(q x_{1}-q_{s a l} x_{b}-q_{2} x_{b}+A v s\left(x_{d}\right)-A v s\left(x_{b}\right)\right)\right| \leq \varepsilon \\
& \left|\frac{d x_{r}}{d t}\right|=\left|\frac{1}{A l_{r}}\left(q_{2} x_{b}-q_{2} x_{r}+A v s\left(x_{b}\right)\right)\right| \leq \varepsilon \\
& \left|\frac{d x_{d}}{d t}\right|=\left|\frac{1}{A l_{d}}\left(q_{s a l} x_{b}-q_{s a l} x_{d}-\operatorname{Avs}\left(x_{d}\right)\right)\right| \leq \varepsilon
\end{aligned}
$$

Controllability constraints:

The controllability constraints are stated to guarantee disturbance rejection capability, either in open or closed loop configurations.

- $\quad$ The ISE norm

$$
I S E=\int_{t=0}^{T \max }\left(s_{1 r}-s_{1}\right)^{2} \cdot d t
$$

where $T_{\max }=165$ hours is the simulation time and $s_{1 r}$ is the steady-state value or reference for substrate.

- The $\mathrm{H}_{\infty}$ norm of the disturbance transfer function:

$$
\left\|G_{d}\right\|_{\infty}=\max _{w} \bar{\sigma}(\omega)
$$

where $\bar{\sigma}$ is the maximum singular value

- The disturbance sensitivity gains at the dominant frequencies of the disturbances.

$$
D s(\omega)=\frac{\left\|G_{d}(j \omega) \cdot d\right\|_{2}}{\|d\|_{2}}
$$

where $G_{d}$ is the disturbance transfer function, $\omega$ is the frequency, and $d$ is the maximum disturbance vector.

\section{SOLVING THE PROBLEM BY DETERMINISTIC AND STOCHASTIC METHODS}

4.1. Solving the problem by deterministic methods: Sequential Quadratic Programming (SQP)

When tackling the Integrated Design mathematical problem, specific features of the process (nonlinearities, different sensitivity for plant parameters and controller parameters, etc.) increase the complexity of the problem. For this reason, when solving closed loop Integrated Design, we propose a methodology consisting of an iterative two steps approach. For open loop design, optimization of function (1) is sufficient, but for closed loop Integrated Design, the optimization procedure involves the two cost functions (1) and $f_{2}$,

$$
f_{2}(x)=w_{5} \cdot I S E
$$

where ISE is obtained by equation (14) and $w_{5}$ is the corresponding weight.

The first step performs the plant design optimizing $f_{1}$, and the second step the controller tuning optimizing $f_{2}$. At every step, plant or controller parameters obtained are used as constant values for the following optimization step. The loop ends when a convergence criterion is reached. (figure 3 )

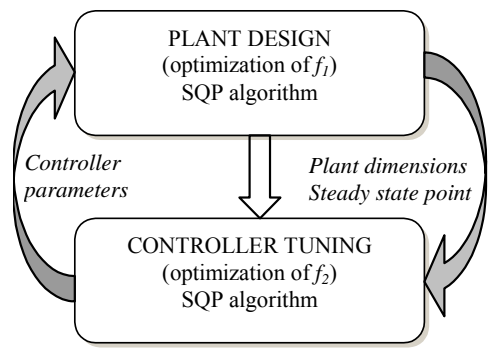

Figure 3: Iterative loop for Integrated Design

For SQP method implementation the MATLAB Optimization Toolbox has been used.

4.2. Solving the problem by stochastic methods: genetic algorithms (GA)

Genetic algorithms are stochastic optimisation techniques based on natural evolution. The optimisation process is carried out with a population of potential solutions for the problem coded as chromosomes (individuals). A fitness value based on the cost estimation is assigned to each one. For each generation, a number of individuals of the population 
are chosen to mate; the selection process favours the best fitted individuals. The mating results in possible new solutions, which can be randomly mutated, and included into population, deleting some previous existing ones. After several generations, the algorithm converges to the best individual, which hopefully represents an optimal solution to the problem (Gen and Chen, 2000).

For this particular case, real coding is proposed, defining each chromosome as $\left[x_{1}, s_{1}, c_{1}, x_{d}, x_{b}, x_{r}, q_{r}\right.$, $\left.q_{p}, f k, V, A\right]$, where the variables are coded as genes in the range [ $\left.\begin{array}{ll}0 & 1\end{array}\right]$. The tournament operator was used for selection procedure. The "arithmetic extended intermediate crossover" is used to generate new candidate solutions. This crossover operator calculates the genes of the offspring $\left(z_{i}\right)$ as the weighted average of the genes of the parents $\left(x_{i}, y_{i}\right)$, as shown in equation (18) (Gen and Chen, 2000).

where

$$
z_{i}=\lambda_{1} \cdot x_{i}+\lambda_{2} \cdot y_{i}
$$

$$
\lambda_{1}+\lambda_{2} \leq 2 \quad \text { (19) } \quad \lambda_{1} \geq 0 \text { (20) } \quad \lambda_{2} \geq 0
$$

It is important to find an appropriated technique to deal with constraints. In this case, an evaluation function with penalty term in the addition form was applied (Gen and Chen, 2000).

For the closed loop design, chromosomes include controller parameters. The algorithm should also favour the exploration of the space of possible controllers for a particular plant, therefore, the population contains individuals representing different plant-controller alternatives and individuals which represent the same plant with different controllers and its size is duplicated.

The optimization problems presented in this work were solved using a population size of 30 individuals and a maximum generation number of 300 .

\subsection{Solving the problem by stochastic methods: simulated annealing (SA)}

Another stochastic method used in this work is the simulated annealing, that is inspired in the annealing process to get minimum energy states in a solid. Mathematically, the states represent candidate solutions, and the energy is the cost associated to each of them. Starting with a random initial point, a sequence of candidate solutions is generated iteratively using the following acceptance criteria. If $i$ is the previous candidate, with cost $f(i)$, and $j$ is a new generated candidate with cost $f(j)$, the acceptance probability of taking $j$ as new candidate solution is

$$
P(\text { accept } j)=\left\{\begin{array}{l}
1 \quad \text { if } f(j) \leq f(i) \\
\exp \left(\frac{f(i)-f(j)}{c}\right) \text { if } f(j)>f(i)
\end{array}\right.
$$

where $c$ is a parameter that decreases when the number of iterations increases. This acceptance probability allows for the algorithm to select solutions that increase cost, in order to avoid local minima.

Candidate solutions are encoded as normalized real number vectors, and the generation of a new candidate consists of changing randomly one of the vector elements (representing one of the optimization variables). Cooling schedule for $c$ parameter used here is a linear decreasing with rate 0.88 .

\subsection{Solving the problem by a hybrid method combining deterministic and stochastic methods}

Stochastic methods have the advantage of avoiding local minima and the ability of providing solutions when dealing with complex problems. If binary or integer variables are involved in the optimization problem (such as process synthesis or model predictive controller parameters tuning), this methods are particularly suitable. However, sometimes they do not arrive to feasible solutions easily, and give only solutions close to an optimum. On the other hand, deterministic methods alone, such as SQP, do not converge to any solution when dealing with complex problems. This is the reason why we propose a combination of both methods, consisting on a final refinement of stochastic solutions with the SQP method. In this way, advantages of both methods are exploited.

\section{INTEGRATED DESIGN RESULTS}

Three scenarios are presented to study the Integrated Design problem and the effectiveness of the algorithms proposed. First, the design was performed to optimise investment and operation costs without any controllability considerations. The second case is focused in open loop design including disturbance sensitivity gains and $\mathrm{H}_{\infty}$ norm as controllability measures. Finally the Integrated Design of the plant with a PI controller is developed. For the three design cases, results with deterministic and stochastic methods are presented, and also results using the hybrid methodology (GA refined) proposed in the paper.

\subsection{Design considering only investment and operation costs}

In this design case, optimum costs plants are obtained, together with a steady state working point. Results are shown in table 1 , and the plant response when disturbances are present is in figure 4 .

Table 1: Integrated Design results without controllability

\begin{tabular}{lcccc}
\hline Parameters & SQP & GA & SA & GA refined \\
\hline$V\left(\mathrm{~m}^{3}\right)$ & 5046 & 5939 & 4829 & 5066 \\
$A\left(\mathrm{~m}^{2}\right)$ & 1885 & 1980 & 1775 & 1887 \\
$S_{1}(\mathrm{mg} / \mathrm{l})$ & 87.5 & 86.21 & 85.30 & 87.47 \\
ISE & 588790 & 528460 & 891970 & 594770 \\
Cost & 0.040 & 0.059 & 0.035 & 0.040 \\
Ds $\left(\omega_{1}\right)$ & 2.342 & 2.203 & 2.324 & 2.340 \\
Ds $\left(\omega_{2}\right)$ & 2.700 & 2.609 & 2.6775 & 2.696 \\
Norm $\mathrm{H}_{\infty}$ & 0.2900 & 0.286 & 0.1609 & 0.2908
\end{tabular}




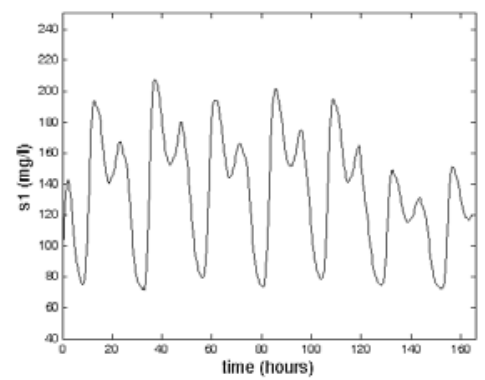

Figure 4: Output substrate of the plant resulting from design without controllability using SQP

\subsection{Design considering open loop controllability constraints}

The aim here is to design an optimum plant that rejects the two load disturbances at the output variable, $s_{1}$, without any controller. The solutions are presented in tables 2 and 3 , and the plant response is shown in figures 5 and 6 . At the view of dynamical responses and controllability indexes, plants obtained in this case exhibit a better disturbance rejection than the economically optimal plants shown in section 5.1, but with higher costs.

Table 2: Open loop Integrated Design results with constraints in disturbance sensitivity gains

\begin{tabular}{lcccc}
\hline Parameters & SQP & GA & SA & GA refined \\
\hline$V\left(\mathrm{~m}^{3}\right)$ & 7270 & 6317 & 6215 & 6762 \\
$A\left(\mathrm{~m}^{2}\right)$ & 2372 & 3075 & 3013 & 2615 \\
$S_{1}(\mathrm{mg} / \mathrm{l})$ & 50.72 & 51.52 & 50.06 & 50.53 \\
$\mathrm{ISE}$ & 186230 & 179110 & 175520 & 184330 \\
Cost & 0.0807 & 0.0985 & 0.0895 & 0.0812 \\
Ds $\left(\omega_{1}\right)$ & 1.354 & 1.378 & 1.379 & 1.366 \\
Ds $\left(\omega_{2}\right)$ & 1.499 & 1.494 & 1.497 & 1.500 \\
Norm $\mathrm{H}_{\infty}$ & 0.151 & 0.156 & 0.160 & 0.1573
\end{tabular}

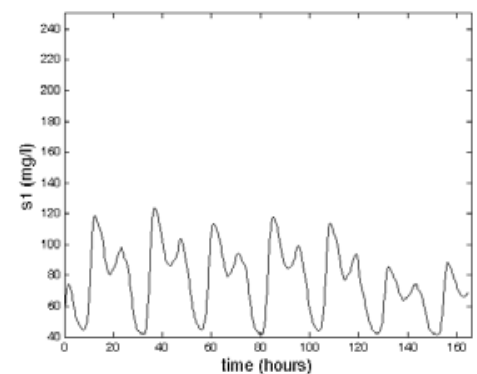

Figure 5: Response of the plant resulting from the Integrated Design considering Ds gains

Table 3: Open loop Integrated Design results with constraint in $\mathrm{H}_{\infty}$ norm

\begin{tabular}{lcccc}
\hline Parameters & SQP & GA & SA & GA refined \\
\hline$V\left(\mathrm{~m}^{3}\right)$ & 7772 & 7784 & 6777 & 5968 \\
$A\left(\mathrm{~m}^{2}\right)$ & 2172 & 2447 & 2165 & 2990 \\
$S_{1}(\mathrm{mg} / \mathrm{l})$ & 51.26 & 51.41 & 51.42 & 51.32 \\
$\mathrm{ISE}$ & 185350 & 158080 & 485110 & 194430 \\
Cost & 0.083 & 0.104 & 0.0706 & 0.0863 \\
Ds $\left(\omega_{1}\right)$ & 1.349 & 1.355 & 1.382 & 1.414 \\
Ds $\left(\omega_{2}\right)$ & 1.510 & 1.508 & 1.574 & 1.537 \\
Norm $\mathrm{H}_{\infty}$ & 0.1600 & 0.1609 & 0.1601 & 0.1600
\end{tabular}

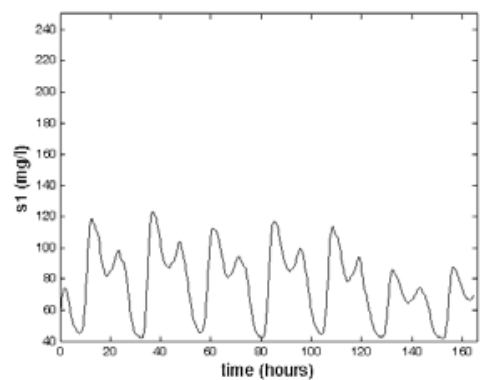

Figure 6: Response of the plant resulting from the Integrated Design considering $\mathrm{H}_{\infty}$ norm

\subsection{Closed loop Integrated Design}

The complexity of the Integrated Design for the closed loop operation increases significantly because now this is a multiobjective dynamical optimisation problem, where the plant and controller parameters $\left(K_{p}, T_{i}\right)$ were determined simultaneously, satisfying the compromise between economics and controllability. Results are presented in table 4 . Now the dynamical performance index ISE is much better than for open loop design. In figures 7 and 8 can be easily seen good disturbance rejection, indicating an appropriated controller tuning.

Table 4: Closed loop Integrated Design results

\begin{tabular}{lccc}
\hline Parameters & SQP & GA & GA refined \\
\hline$V\left(\mathrm{~m}^{3}\right)$ & 8570 & 9664 & 8611 \\
$A\left(\mathrm{~m}^{2}\right)$ & 3084 & 2405 & 3026.1 \\
$S_{1}(\mathrm{mg} / \mathrm{l})$ & 47.38 & 59.00 & 38.63 \\
$\mathrm{ISE}$ & 79791 & 75114 & 79771 \\
Cost & 0.1335 & 0.1355 & 0.1292 \\
$K_{p}$ & -11.94 & -41.10 & -7.33 \\
$T_{i}$ & 0.1330 & 626.7 & 415.1 \\
${\text { Norm } \mathrm{H}_{\infty}}^{0.4220}$ & 0.1274 & 0.1080 \\
\hline
\end{tabular}

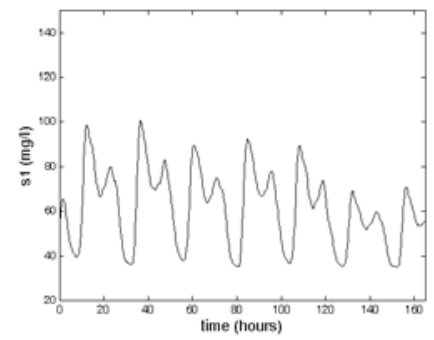

Figure 7: Response of the plant resulting from the closed loop Integrated Design with SQP

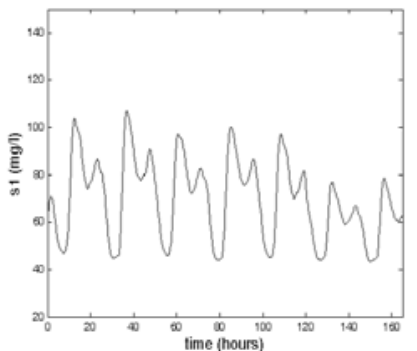

Figure 8: Response of the plant resulting from the closed loop Integrated Design with GA 
In general, the effectiveness of the algorithms applied to solve the Integrated Design problem is satisfactory in all the cases studied. For the open loop design, the SQP method exhibited better performance in terms of computational time and solutions quality. The stochastic algorithms always lead to solutions comparable to the SQP results, but when dealing with constraints these methods provide frequently non feasible solutions, especially when using simulated annealing. However, the use of refined GA with SQP gives quite good solutions with a reasonable computational effort.

The closed loop design was solved using only SQP, GA and the hybrid methodology. The computational time required to solve the problem was similar for both techniques, and different results with similar quality were found. It is important to note that, for this complex case, the hybrid methodology gives the best solution.

\section{CONCLUSIONS}

In this paper different optimization methods have been studied to solve the Integrated Design problem of processes, and particularly the activated sludge process. Two stochastic methods (simulated annealing and genetic algorithms) and one deterministic (sequential quadratic programming) have been applied, showing good results in open loop design and closed loop Integrated Design with PI controllers.

Furthermore, hybrid optimization starting with GA and refining solutions with SQP has also been developed, combining advantages of both methods, and giving also good results for Integrated Design. Because of GA seems very suitable for solving MINLP problems, these results are encouraging for the application of the hybrid method to solve the problems derived from process synthesis, or Integrated Design with model predictive controllers, that also involves integer variables.

Note that plant non-linear dynamics has also been considered in the Integrated Design process. Plants designed are optimum costs and feature optimum disturbance rejection depending on requirements. The non-linear model of the plant is satisfied, as well as the operation and process constraints.

\section{Acknowledgments}

The authors gratefully acknowledge the support of the Spanish Government through the CICYT project DPI2003-09392-C02-02. Co-authors S. Revollar and $R$. Lamanna also acknowledge support of the Venezuelan funding institution FONACIT, under grant No. S1-99000111

\section{REFERENCES}

Costa, L. and Oliveira, P. (2001). Evolutionary Algorithms Approach to the Solution of Mixed Integer Non-Linear Programming Problems.
Comp. Chem. Eng., 25 257-266

Edgar, T. and Himmelblau, D. (2001) Optimization of Chemical Processes. Mc Graw Hill

Gen, M. and Chen, R. (2000). Genetic algorithms and engineering optimisation. John Wiley and Sons.

Gill, P., Murray, W. and Wright, M. (1981) Practical Optimization. Academic Press.

Kookos I. and Perkins J. (2001). An algorithm for simultaneous process design and control. Ind. Eng. Chem. Res., 40, pp. 4079-4088.

Laarhoven, P and Aarts, E. (1987) Simulated Annealing: Theory and Applications. Kluwer Academic Publishers

Luyben, M. L. and Floudas, C. A (1994). Analyzing the interaction of design and control -1. A multiobjective framework and application to binary distillation synthesis, Comp. Chem. Eng., 18, No. 10, pp. 933-969.

Luyben, W. (1993). Trade-offs Betwen Design and Control in Chemical Reactor Systems. J. Proc. Cont. 3, pp. 17-40.

Revollar, S; Vega, P. and Lamanna, R. (2004) Algorithmic synthesis and Integrated Design of chemical reactor systems using genetic algorithms. World Automation Congress (Sevilla, Spain).

Salamon, P. Sibani, P. and Frost, R. (2002). Facts, Conjectures, and Improvements for Simulated Annealing. SIAM monographs on Mathematical Modelling and Computation.

Tsai, M. and Chang, Ch. (2001). Water usage and treatment network design using genetic algorithms Ind. Eng. Chem. Res., 40, pp. 48744888.

Vega, P. and Gutiérrez, G. (1999). Optimal Design Control and Operation of wastewater treatment plants. European Control Conference (Germany). 